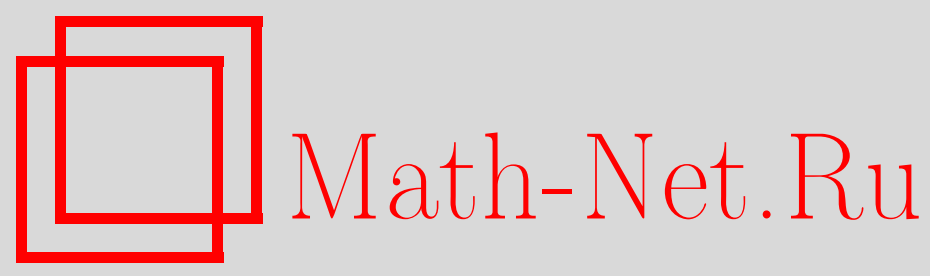

Н. М. Добровольский, А. Л. Рощеня, И. Ю. Реброва, Непрерывность гиперболической дзета-функции решеток, $M a$ тем. заметки, 1998, том 63, выпуск 4, 522-526

DOI: https://doi.org/10.4213/mzm1312

Использование Общероссийского математического портала Math-Net.Ru подразумевает, что вы прочитали и согласны с пользовательским соглашением http://www . mathnet.ru/rus/agreement

Параметры загрузки:

IP: 54.196 .121 .252

26 апреля 2023 г., 12:37:03

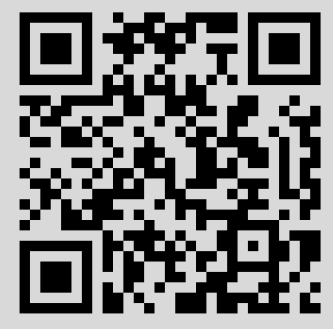




\title{
НЕПРЕРЫВНОСТЬ ГИПЕРБОЛИЧЕСКОЙ ДЗЕТА-ФУНКЦИИ РЕШЕТОК
}

\author{
Н. М. Добровольский, А. Л. Рощеня, И. Ю. Реброва
}

\begin{abstract}
Доказана непрерывность гиперболической дзета-функции решеток $\zeta_{H}(\Lambda \mid \alpha)$, $\alpha=\sigma+i t, \sigma>1$, как функции на метрическом пространстве решеток.

Библиограффия: 2 названия.
\end{abstract}

Пусть $s \geqslant 2$ и полная решетка $\Lambda \subset \mathbb{R}^{s}$. Тогда гиперболической дзета-функиией решетки $\Lambda$ назьвается

$$
\zeta_{H}(\Lambda \mid \alpha)=\sum_{\vec{x} \in \Lambda}^{\prime}\left(\bar{x}_{1} \cdots \bar{x}_{s}\right)^{-\alpha}
$$

где $\sum^{\prime}$ означает, что из области суммирования исключена нулевая точка решетки $\Lambda$, и $\bar{x}=\max \{1,|x|\}$ для любого действительного $x$.

Гиперболическая дзета-функция решетки является аналитической функцией комплексной переменной $\alpha=\sigma+i t$ в правой полуплоскости $\sigma>1$.

Как известно (см. [1, с. 165]), пространство решеток является метрическим пространством. Возникает естественньй вопрос о поведении гиперболической дзета-функции решеток как функции от решеток.

Цель настоящей работы - доказать непрерывность гиперболической дзета-функции решеток на пространстве решеток. В частности, справедлив следуюший основной результат.

Теорема. Если последовательность решеток $\left\{\Lambda_{n}\right\}$ сходится $\propto$ решетке $\Lambda$, то последовательность гиперболических дзета-функиий решеток $\zeta_{H}\left(\Lambda_{n} \mid \alpha\right)$ равномерно сходится $к$ гиперболической дзета-функиии решетки $\zeta_{H}(\Lambda \mid \alpha)$ в любой полуплоскости $\sigma \geqslant \sigma_{0}>1$.

1. Обозначения и вспомогательные результаты. Гиперболическим крестом назьвается область

$$
K(T)=\left\{\vec{x} \mid \bar{x}_{1} \cdots \bar{x}_{s} \leqslant T\right\} .
$$

Пусть $D(T \mid \Lambda)$ - количество ненулевых точек решетки $\Lambda$ в гиперболическом кресте $K(T)$. Рассмотрим произвольньй базис решетки $\Lambda: \vec{\lambda}_{j}=\left(\lambda_{j 1}, \ldots, \lambda_{j s}\right), j=1, \ldots, s$, и величину

$$
A\left(\vec{\lambda}_{1}, \ldots, \vec{\lambda}_{s}\right)=\frac{1}{2} \max _{1 \leqslant j \leqslant s} \sum_{\nu=1}^{s}\left|\lambda_{\nu j}\right| .
$$

(C) Н. М. Довровольский, А. Л. РОшеня, И. Ю. РевровА 
Определим величину

$$
a(\Lambda)=\min _{\vec{\lambda}_{1}, \ldots, \vec{\lambda}_{s}} \max \left\{1, A\left(\vec{\lambda}_{1}, \ldots, \vec{\lambda}_{s}\right)\right\}
$$

где минимум берется по всем базисам $\vec{\lambda}_{1}, \ldots, \vec{\lambda}_{s}$ решетки $\Lambda$.

В работе [2] доказан следующий результат.

ТЕОРемА А. При $T \geqslant 3$ справедлива асимптотическая формула

$$
D(T \mid \Lambda)=\frac{2^{s} T \ln ^{s-1} T}{(s-1) ! \operatorname{det} \Lambda}+\frac{\Theta \cdot 4 \cdot 2^{s}(a(\Lambda)+2)^{s} T \ln ^{s-2} T}{\operatorname{det} \Lambda}
$$

əде $|\Theta| \leqslant 1$.

Известно (см. [1, с. 160-165]), что если последовательность решеток $\left\{\Lambda_{n}\right\}$ сходится к решетке $\Lambda$, то существует последовательность невырожденных матриц

$$
A_{n}=\left(\begin{array}{ccc}
a_{11}^{(n)} & \ldots & a_{1 s}^{(n)} \\
\vdots & \ddots & \vdots \\
a_{s 1}^{(n)} & \ldots & a_{s s}^{(n)}
\end{array}\right), \quad n=1,2, \ldots, \quad \operatorname{det} \Lambda_{n} \neq 0
$$

сходящаяся к единичной матрице

$$
E=\left(\begin{array}{ccc}
\delta_{11} & \ldots & \delta_{1 s} \\
\vdots & \ddots & \vdots \\
\delta_{s 1} & \ldots & \delta_{s s}
\end{array}\right), \quad \text { где } \delta_{i j}=\left\{\begin{array}{cc}
1 & \text { при } i=j \\
0 & \text { при } i \neq j .
\end{array}\right.
$$

Иными словами,

$$
\left\|A_{n}-E\right\|=s \max _{1 \leqslant i, j \leqslant s}\left|a_{i j}^{(n)}-\delta_{i j}\right| \rightarrow 0 \quad \text { при } n \rightarrow \infty,
$$

и, кроме того, $\Lambda_{n}=A_{n} \Lambda$.

Лемма 1. Если $\Lambda_{n} \rightarrow \Lambda$ прu $n \rightarrow \infty$, mo $a\left(\Lambda_{n}\right) \rightarrow a(\Lambda)$ npu $n \rightarrow \infty$.

ДокАЗАТЕЛЬСТво. Из условия следует, что найдется последовательность матрищ $\left\{A_{n}\right\}$ такая, что

$$
\Lambda_{n}=A_{n} \Lambda, \quad\left\|A_{n}-E\right\| \rightarrow 0 \quad \text { при } n \rightarrow \infty .
$$

Положим $\vec{\lambda}_{j}^{(n)}=A_{n} \vec{\lambda}_{j}, j=1, \ldots, s$. Тогда в силу (1)

$$
\lim _{n \rightarrow \infty} A\left(\vec{\lambda}_{1}^{(n)}, \ldots, \vec{\lambda}_{s}^{(n)}\right)=A\left(\vec{\lambda}_{1}, \ldots, \vec{\lambda}_{s}\right)
$$

Выбрав базис $\vec{\lambda}_{1}, \ldots, \vec{\lambda}_{s}$ решетки $\Lambda$ согласно условию

$$
a(\Lambda)=\max \left\{1, A\left(\vec{\lambda}_{1}, \ldots, \vec{\lambda}_{s}\right)\right\}
$$

из (2) получим, что справедливо неравенство

$$
\varlimsup_{n \rightarrow \infty} a\left(\Lambda_{n}\right) \leqslant a(\Lambda)
$$


С другой стороны, положим

$$
a_{0}=\lim _{n \rightarrow \infty} a\left(\Lambda_{n}\right)
$$

и выберем базисы $\vec{\lambda}_{1}^{\left(n_{j}\right)}, \ldots, \vec{\lambda}_{s}^{\left(n_{j}\right)}$ решеток $\Lambda_{n_{j}}$ согласно условию

$$
a\left(\Lambda_{n_{j}}\right)=\max \left\{1, A\left(\vec{\lambda}_{1}^{\left(n_{j}\right)}, \ldots, \vec{\lambda}_{s}^{\left(n_{j}\right)}\right)\right\}, \quad \lim _{j \rightarrow \infty} a\left(\Lambda_{n_{j}}\right)=a_{0}
$$

Тогда, в силу ограниченности модуля координат сверху для всех векторов базиса $\vec{\lambda}_{1}^{\left(n_{j}\right)}$, $\ldots, \vec{\lambda}_{s}^{\left(n_{j}\right)}$ при $j \rightarrow \infty$, по теореме Вейерштрасса можно выделить подпоследовательность, сходящуюся к некоторому базису $\vec{\lambda}_{1}^{\prime}, \ldots, \vec{\lambda}_{s}^{\prime}$ решетки $\Lambda$. И так как

$$
\lim _{j \rightarrow \infty} A\left(\vec{\lambda}_{1}^{\left(n_{j}\right)}, \ldots, \vec{\lambda}_{s}^{\left(n_{j}\right)}\right)=A\left(\vec{\lambda}_{1}^{\prime}, \ldots, \vec{\lambda}_{s}^{\prime}\right)
$$

имеем $a_{0} \geqslant a(\Lambda)$.

Отсюда вытекает

$$
\varlimsup_{n \rightarrow \infty} a\left(\Lambda_{n}\right)=\underline{\lim }_{n \rightarrow \infty} a\left(\Lambda_{n}\right)=\lim _{n \rightarrow \infty} a\left(\Lambda_{n}\right)=a(\Lambda)
$$

что и требовалось доказать.

ЛЕмма 2. Для $\alpha=\sigma+i t$ при $\sigma \geqslant \sigma_{0}>1, T \geqslant 3$ справедлива приближсенная формула

$$
\zeta_{H}(\Lambda \mid \alpha)=\sum_{\vec{x} \in \Lambda \cap K(T)}^{\prime}\left(\bar{x}_{1} \cdots \bar{x}_{s}\right)^{-\alpha}+\frac{\Theta_{1}(\alpha, T) c_{1}(s)}{\operatorname{det} \Lambda \cdot T^{\sigma_{0}-1}} \frac{\sigma_{0}}{\sigma_{0}-1}\left(\frac{1}{\left(\sigma_{0}-1\right)^{s-1}}+\ln ^{s-1} T\right)
$$

әде $\left|\Theta_{1}(\alpha, T)\right| \leqslant 1, c_{1}(s)=2^{s} \cdot 24(s-2) !(a(\Lambda)+2)^{s}$.

ДокАзАТЕЛЬСТво. Представим дзета-ряд для гиперболической дзета-функции решетки $\Lambda$ в виде

$$
\zeta_{H}(\Lambda \mid \alpha)=\sum_{\vec{x} \in \Lambda \cap K(T)}^{\prime}\left(\bar{x}_{1} \cdots \bar{x}_{s}\right)^{-\alpha}+R(\Lambda, T \mid \alpha)
$$

где

$$
R(\Lambda, T \mid \alpha)=\sum_{\substack{\vec{x} \in \Lambda \\ \bar{x}_{1} \cdots \bar{x}_{s}>T}}\left(\bar{x}_{1} \cdots \bar{x}_{s}\right)^{-\alpha}
$$

Оценивая по модулю каждое слагаемое в (3), получим $|R(\Lambda, T \mid \alpha)| \leqslant R(\Lambda, T \mid \sigma) \leqslant$ $R\left(\Lambda, T \mid \sigma_{0}\right)$. По теореме Абеля

$$
R\left(\Lambda, T \mid \sigma_{0}\right)=\sigma_{0} \int_{T}^{\infty} \frac{D(x \mid \Lambda)-D(T \mid \Lambda)}{x^{\sigma_{0}+1}} d x
$$


Отсюда и из теоремы А следует

$$
\begin{aligned}
R\left(\Lambda, T \mid \sigma_{0}\right) \leqslant & \sigma_{0} \int_{T}^{\infty}\left(\frac{2^{s} x \ln ^{s-1} x}{(s-1) ! \operatorname{det} \Lambda}+\frac{2^{s+2}(a(\Lambda)+2)^{s} x \ln ^{s-2} x}{\operatorname{det} \Lambda}\right) \frac{d x}{x^{\sigma_{0}+1}} \\
= & \frac{2^{s} \sigma_{0}}{\operatorname{det} \Lambda}\left(\frac{1}{(s-1) !} \int_{T}^{\infty} \frac{\ln ^{s-1} x}{x^{\sigma_{0}}} d x+4(a(\Lambda)+2)^{s} \int_{T}^{\infty} \frac{\ln ^{s-2} x}{x^{\sigma_{0}}} d x\right) \\
= & \frac{2^{s} \sigma_{0}}{\operatorname{det} \Lambda}\left(\frac{1}{(s-1) ! T^{\sigma_{0}-1}} \sum_{k=0}^{s-1} \frac{(s-1) !}{\left(\sigma_{0}-1\right)^{s-k} k !} \ln ^{k} T\right. \\
& \left.+\frac{4(a(\Lambda)+2)^{s}}{T^{\sigma_{0}-1}} \sum_{k=0}^{s-2} \frac{(s-2) !}{\left(\sigma_{0}-1\right)^{s-1-k} k !} \ln ^{k} T\right) \\
\leqslant & \frac{2^{s} \sigma_{0}}{\operatorname{det} \Lambda \cdot T^{\sigma_{0}-1}}\left(3\left(\frac{1}{\left(\sigma_{0}-1\right)^{s}}+\frac{\ln ^{s-1} T}{\sigma_{0}-1}\right)\right. \\
& \left.+12(s-2) !(a(\Lambda)+2)^{s}\left(\frac{1}{\left(\sigma_{0}-1\right)^{s-1}}+\frac{\ln ^{s-2} T}{\sigma_{0}-1}\right)\right) \\
\leqslant & \frac{2^{s} \cdot 24(s-2) !(a(\Lambda)+2)^{s}}{\operatorname{det} \Lambda \cdot T^{\sigma_{0}-1}} \frac{\sigma_{0}}{\sigma_{0}-1}\left(\frac{1}{\left(\sigma_{0}-1\right)^{s-1}}+\ln ^{s-1} T\right) .
\end{aligned}
$$

Лемма доказана.

2. Доказательство основной теоремы. Пусть $\Lambda_{0}=\Lambda$. Определим величины

$$
a=\max _{n \geqslant 0} a\left(\Lambda_{n}\right), \quad b=\min _{n \geqslant 0} \operatorname{det} \Lambda_{n}
$$

Ясно, что $b>0$. И так как последовательность $\left\{\Lambda_{n}\right\}$ сходящаяся, то из леммы 1 вытекает, что величина $a$ конечная. Выберем $T_{1}=T_{1}(\varepsilon)$ из условия

$$
\frac{2^{s} \cdot 24(s-2) !(a+2)^{s}}{b T_{1}^{\sigma_{0}-1}} \frac{\sigma_{0}}{\sigma_{0}-1}\left(\left(\sigma_{0}-1\right)^{-s+1}+\ln ^{s-1} T_{1}\right)<\frac{\varepsilon}{3} .
$$

Тогда при $T \geqslant T_{1}$ для любого $n \geqslant 0$ имеем

$$
\left|R\left(\Lambda_{n}, T \mid \alpha\right)\right| \leqslant R\left(\Lambda_{n}, T_{1} \mid \sigma_{0}\right)<\frac{\varepsilon}{3}
$$

в полуплоскости $\alpha=\sigma+i t, \sigma \geqslant \sigma_{0}>1$. Рассмотрим крест $K\left(2 T_{1}\right)$ и все ненулевые точки решетки $\Lambda$, принадлежашие этому гиперболическому кресту. Пусть это точки $\vec{x}_{1}, \ldots, \vec{x}_{N}$, где $N=D\left(2 T_{1} \mid \Lambda\right)$.

Для сходящейся к единичной матрице $E$ последовательности матриц $\left\{A_{n}\right\}$, определенной условиями

$$
\Lambda_{n}=A_{n} \Lambda, \quad \lim _{n \rightarrow \infty} A_{n}=E,
$$

рассмотрим точки $\vec{y}_{1}^{(n)}=A_{n} \vec{x}_{1}, \ldots, \vec{y}_{N}^{(n)}=A_{n} \vec{x}_{N}$ из решетки $\Lambda_{n}$. В силу сходимости

$$
\lim _{n \rightarrow \infty} A_{n} \vec{x}_{j}=\vec{x}_{j}, \quad j=1, \ldots, N,
$$


найдется $n_{0}=n_{0}(\varepsilon)$ такое, что для любого $n \geqslant n_{0}$ все точки $\vec{y}_{j}^{(n)}$, для которых $\vec{x}_{j} \in K\left(T_{1}\right)$, принадлежат кресту $K\left(2 T_{1}\right)$ и каждая точка $\vec{y}_{j}^{(n)}$, принадлежащая кресту $K\left(T_{1}\right)$, имеет прообраз $\vec{x}_{j}$, принадлежащий кресту $K\left(2 T_{1}\right)$.

Отсюда следует, что для $\alpha=\sigma+i t, \sigma \geqslant \sigma_{0}$,

$$
\begin{aligned}
& \left|\zeta_{H}\left(\Lambda_{n} \mid \alpha\right)-\zeta_{H}(\Lambda \mid \alpha)\right| \\
& \leqslant\left|\zeta_{H}\left(\Lambda_{n} \mid \alpha\right)-\sum_{j=1}^{N}\left(\bar{y}_{j 1}^{(n)} \cdots \bar{y}_{j s}^{(n)}\right)^{-\alpha}\right| \\
& \quad+\left|\sum_{j=1}^{N}\left(\bar{y}_{j 1}^{(n)} \cdots \bar{y}_{j s}^{(n)}\right)^{-\alpha}-\sum_{j=1}^{N}\left(\bar{x}_{j 1} \cdots \bar{x}_{j s}\right)^{-\alpha}\right|+\left|\zeta_{H}(\Lambda \mid \alpha)-\sum_{j=1}^{N}\left(\bar{x}_{j 1} \cdots \bar{x}_{j s}\right)^{-\alpha}\right| \\
& \quad \leqslant R\left(\Lambda_{n}, T_{1} \mid \sigma_{0}\right)+R\left(\Lambda, T_{1} \mid \sigma_{0}\right)+\sum_{j=1}^{N}\left|\left(\bar{y}_{j 1}^{(n)} \cdots \bar{y}_{j s}^{(n)}\right)^{-\sigma_{0}}-\left(\bar{x}_{j 1} \cdots \bar{x}_{j s}\right)^{-\sigma_{0}}\right| \\
& \quad \leqslant \frac{2 \varepsilon}{3}+\sum_{j=1}^{N} \mid\left(\bar{y}_{j 1}^{(n)} \cdots \bar{y}_{j s}^{(n)}\right)^{-\sigma_{0}}-\left(\bar{x}_{j 1} \cdots \bar{x}_{j s}\right)^{-\sigma_{0} \mid .}
\end{aligned}
$$

Выбирая $n_{1}=n_{1}(\varepsilon)$ из условия

$$
\left|\left(\bar{y}_{j 1}^{(n)} \cdots \bar{y}_{j s}^{(n)}\right)^{-\sigma_{0}}-\left(\bar{x}_{j 1} \cdots \bar{x}_{j s}\right)^{-\sigma_{0}}\right| \leqslant \frac{\varepsilon}{3 N}
$$

при $n \geqslant n_{1}$, получим $\left|\zeta_{H}\left(\Lambda_{n} \mid \alpha\right)-\zeta_{H}(\Lambda \mid \alpha)\right| \leqslant \varepsilon$ для любого $n \geqslant \max \left\{n_{0}(\varepsilon), n_{1}(\varepsilon)\right\}$.

Теорема полностью доказана.

Авторы выражают благодарность профессору Н. М. Коробову и профессору В. И. Нечаеву за полезные обсуждения.

\section{СПИСОК ЦИТИРОВАННОЙ ЛИТЕРАТУРЫ}

[1] Касселс Дж. Введение в геометрию чисел. М.: Мир, 1965.

[2] Добровольский Н. М., Рощеня А. Л. О числе точек решетки в гиперболическом кресте // Матем. заметки. 1998. Т. 63. № 3. С. 363-369. 